\title{
ON THE CIRCLE METHOD OF SUMMATION OF A CAUCHY PRODUCT SERIES ${ }^{1}$
}

\section{KAZUO ISHIGURO}

1. Concerning the Euler summability of a Cauchy product series Knopp $[1 ; 2]$ proved the theorems of Abel's and Mertens' type, and later Hara [3] proved the theorem of Cauchy's type. Here the Euler means of a sequence $\left\{s_{n}\right\}$ depend on a parameter $r$, and are defined by the transform

$$
\sigma_{n}(r)=\sigma_{n}=\sum_{\nu=0}^{n}\left(\begin{array}{l}
n \\
\nu
\end{array}\right) r^{\nu}(1-r)^{n-v} s_{\nu}, \quad n=0,1,2, \cdots .
$$

We assume $0<r \leqq 1$, in which case the summation method is regular [2]. The case $r=1$ corresponds to the ordinary convergence. We denote this method of summation by $(\epsilon, r)$.

On the other hand the circle means of a sequence $\left\{s_{n}\right\}$ depend on a parameter $r$ and are defined by the transform

$$
\sigma_{n}^{*}(r)=\sigma_{n}^{*}=\sum_{\nu=n}^{\infty}\left(\begin{array}{l}
\nu \\
n
\end{array}\right) r^{n+1}(1-r)^{\nu-n} s_{\nu}, \quad n=0,1,2, \cdots .
$$

Here we assume $0<r \leqq 1$, in which case the summation is regular also. We denote this method of summation by $(\gamma, r)$.

The relations between $(\epsilon, r)$-method and $(\gamma, r)$-method are studied by Meyer-König [4], Vermes $[5 ; 6]$, Ramanujan $[7 ; 8]$ and the author [9].

The purpose of this short note is to show that the theorems of Abel's and Mertens' and Cauchy's type all hold for the $(\gamma, r)$-method of summation.

2. When a series $\sum_{n=0}^{\infty} a_{n}$ is given, let $s_{n}, n=0,1,2, \cdots$, be the partial sums of this series. If the sequence $\left\{s_{n}\right\}$ is summable by $(\gamma, r)$-method to $A$, then we shall say that

$$
\sum_{n=0}^{\infty} a_{n}=A(\gamma, r) .
$$

This is the same as

Received by the editors August 28, 1961.

${ }^{1}$ This note was presented at the annual meeting of the Mathematical Society of Japan, May 31, 1962 in Tokyo. 


$$
\sum_{n=0}^{\infty} a_{n}^{*}=A
$$

where

$$
a_{n}^{*}=\sum_{n=n}^{\infty}\left(\begin{array}{l}
\nu \\
n
\end{array}\right) r^{n}(1-r)^{p-n} a_{v}, \quad n=0,1,2, \cdots \text { (see [2]). }
$$

If $\sum a_{n}^{*}$ converges absolutely to $A$, then we shall say that

$$
\sum a_{n}=A(|\gamma, r|) \text {. }
$$

Furthermore if series $\sum a_{n}$ and $\sum b_{n}$ are given, then we shall denote that

$$
c_{p}=\sum_{m+n=p} a_{m} b_{n}, \quad \quad p=0,1,2, \cdots
$$

Here we have the next four theorems.

Theorem 1. If $\sum a_{n}=A(\gamma, r), \sum b_{n}=B(\gamma, r)$ and $\sum c_{n}=C(\gamma, r)$, then

$$
A B=C .
$$

THEOREM 2. If $\sum a_{n}=A(|\gamma, r|)$ and $\sum b_{n}=B(\gamma, r)$, then

$$
\sum c_{n}=C(\gamma, r), \text { and } C=A B \text {. }
$$

THEOREM 3. If $\sum a_{n}=A(|\gamma, r|)$ and $\sum b_{n}=B(|\gamma, r|)$, then

$$
\sum c_{n}=C(|\gamma, r|) \text {, and } C=A B \text {. }
$$

TheOREM 4. If $\sum a_{n}=A(\gamma, r)$ and $\sum b_{n}=B(\gamma, r)$, then

$$
\sum c_{n}=A B(\gamma, r ; C, 1)
$$

in the sense that the $(\gamma, r)$ transform of $\sum c_{n}$ is Cesàro $(C, 1)$ summable to $A B$.

Proof. Let

$$
f(x)=\sum_{m=0}^{\infty} a_{m} x^{m}
$$

and $x=1-r+r y ;$ then

$$
f(x)=\sum_{m=0}^{\infty} a_{m}(1-r+r y)^{m}=\sum_{m=0}^{\infty} \frac{f^{(m)}(1-r)}{m !}(r y)^{m}=\sum_{m=0}^{\infty} a_{m}^{*} y^{m},
$$


since

$$
a_{m}^{*}=\frac{f^{(m)}(1-r)}{m !} r^{m}
$$

Similarly we get

$$
\begin{aligned}
& g(x)=\sum b_{n} x^{n}=\sum b_{n}^{*} y^{n} \\
& h(x)=\sum c_{p} x^{p}=\sum c_{p}^{*} y^{p}
\end{aligned}
$$

and

$$
f(x) g(x)=h(x),
$$

for small $x$ and $y$, (see [2]). Hence

$$
\sum_{m+n=p} a_{m}^{*} b_{n}^{*}=c_{p}^{*}
$$

On the other hand for the series $\sum a_{m}^{*}, \sum b_{n}^{*}$ and $\sum c_{p}^{*}$ we know the classical Abel, Mertens, Cauchy theorems and so on, (see [2, Theorems $160,161,162,184])$.

Thus the proofs of Theorem 1, 2 and 3 are complete.

Professor M. S. Ramanujan advised me to add Theorem 4. I wish to express my hearty thanks to him.

\section{REFERENCES}

1. K. Knopp, Über das Eulersche Summierungsverfahren, Math. Z. 18 (1923), 125156.

2. G. H. Hardy, Divergent series, Oxford, at the Clarendon Press, 1949.

3. H. Hara, On the Cauchy's product series theorem on Euler's summability, Kodai Math. Sem. Rep. (1953), 91-92.

4. W. Meyer-König, Untersuchungen über einige verwandte Limitierungsverfahren, Math. Z. 52 (1949), 257-304.

5. P. Vermes, Series to series transformations and analytic continuation by matrix methods, Amer. J. Math. 71 (1949), 541-562.

6. - The transpose of a summability matrix, Colloque sur la theorie des suites (Bruxelles, 1957), pp. 60-86, Gauthier-Villars, Paris, 1958.

7. M. S. Ramanujan, Series to series quasi-Hausdorff transformations, J. Indian Math. Soc. 17 (1953), 47-53.

8. - A note on quasi-Hausdorff series-to-series transformations, J. London Math. Soc. 32 (1957), 27-32.

9. K. Ishiguro, Zur Gibbsschen Erscheinung filr das Kreisverfahren, Math. Z. 76 (1961), 288-294.

HOKKAIDO-UNIVERSITY, JAPAN 